\title{
The Cavity Structure Effect in Medicine: The Physical Aspect
}

\author{
Liudmila B. Boldyreva \\ State University of Management, Moscow, Russia
}

\begin{abstract}
Keywords
Cavity structure effect - Alternative medicine - Model of superfluid physical vacuum - Spin supercurrents

Spin structures in superfluid physical vacuum
\end{abstract}

\section{Summary}

Background: In 1893 the German scientist Oskar Korschelt was granted a patent for using cavity structures (CS) for therapeutic purposes. In the end of the 20th century in Russia Victor Grebennikov also studied possibilities of using CS in curing various diseases. Korschelt used artificial CSs made of copper chains or plates. Grebennikov used mainly natural CS, e.g., empty bee combs. The main features of the CS effect are: the therapeutic efficacy of a CS depends on its orientation with respect to the biological object and on the material the CS is made of. It does not depend on the presence of acoustic or electromagnetic screens. The CS influence on the target biological object may not cease after removal of the CS, i.e. there is an after-effect. Methods: It is shown in the paper that it is possible to explain the effects of CSs on biological objects under the assumption that the physical vacuum has the properties of superfluid ${ }^{3} \mathrm{He}-\mathrm{B}$. Results: The effects may be due to spin supercurrents being present in CSs. Conclusion: CSs differ from bodies of other form in that the cavity is 'filled' with spin supercurrents. The properties of the supercurrents are like those of the spin supercurrents between spin structures in superfluid ${ }^{3} \mathrm{He}-\mathrm{B}$.

\author{
Schlüsselwörter \\ Höhlenstruktureffekt · Alternative Medizin · Modell des \\ suprafluiden physischen Vakuums S Spinströme . \\ Spinstrukturen im suprafluiden physischen Vakuum
}

\section{Zusammenfassung}

Hintergrund: Der deutsche Wissenschaftler Oskar Korschelt bekam 1893 das Patent für die Verwendung von Höhlenstrukturen (HS) zu medizinischen Zwecken. Ende des 20. Jahrhunderts untersuchte Victor Grebennikov in Russland ebenfalls die Nutzungsmöglichkeiten von HS für die Behandlung verschiedener Krankheiten. Korschelt stellte die künstlich erzeugten HS in Form von kupfernen aufeinanderfolgenden Ketten her. Grebennikov dagegen verwendete HS natürlicher Herkunft wie z.B. Bienenwaben. Die Haupteigenschaften von HS-Effekten sind: die therapeutische Wirksamkeit einer HS ist abhängig von der HS-Orientierung in Bezug auf das biologische Objekt und vom Material, aus dem die HS besteht. Sie ist unabhängig vom Vorhandensein akustischer und elektromagnetischer Schutzschirme. Der Einfluss, den eine HS auf das biologische Zielobjekt hat, lässt nicht nach, wenn die HS entfernt wird, d.h. ein Nacheffekt bleibt bestehen. Methoden: In der vorliegenden Arbeit werden Effekte von HS auf biologische Objekte unter der Annahme erklärt, dass das physische Vakuum Eigenschaften von suprafluiden ${ }^{3} \mathrm{He}-\mathrm{B}$ hat. Ergebnisse: Diese Besonderheiten können durch die supraflüssigen Spinströme bedingt sein, die in den HS vorhanden sind. Schlussfolgerung: Die HS unterscheiden sich von anderen Formen dadurch, dass die Höhle mit supraflüssigen Spinströmen und der mit ihnen zusammenhängenden Energie "ausgefüllt» ist. Die Eigenschaften dieser Ströme sind denen der supraflüssigen Spinströme ähnlich, die zwischen den Spinstrukturen im supraflüssigen ${ }^{3} \mathrm{He}-\mathrm{B}$ entstehen.

\begin{tabular}{ll}
\hline KARGER & $\begin{array}{l}\text { (C) 2013 S. Karger GmbH, Freiburg } \\
\text { 1661-4119/13/0205-0322\$38.00/0 }\end{array}$ \\
$\begin{array}{l}\text { Fax +497614520714 } \\
\text { Information@Karger.com }\end{array}$ & $\begin{array}{l}\text { This is an Open Access article licensed under the terms of the } \\
\text { www.karger.com }\end{array}$ \\
$\begin{array}{l}\text { Creative Commons Attribution-NonCommercial 3.0 Unported license } \\
\text { (CC BY-NC) (www.karger.com/OA-license), applicable to the online } \\
\text { version of the article only. Distribution permitted for non-commercial } \\
\text { purposes only. }\end{array}$
\end{tabular}




\section{Introduction}

At present there is much evidence of specific influence exerted on a biological system by ambient bodies having a cavity structure (empty bee combs, meshy structures, porous materials, bundles of tubes, pyramids, and many others).

For example, the book [1] by Manfred Dimde is devoted to healing properties of pyramids. As the results of the experiments conducted by De Belizal and Morel [2] have shown, the germinating ability of seeds changes when the seeds are placed in a pyramid. The studies by De Belizal and Morel as well as Pagot $[2,3]$ describe the experiments with coaxially joined hollow-wooden hemispheres. Placing 9 such hemispheres of $9 \mathrm{~cm}$ in diameter above pieces of fish or meat resulted in their mummification in less than a calendar day. Seven hemispheres of $25 \mathrm{~cm}$ in diameter that were placed above rats in a cage caused panic among them, followed by a kind of catalepsy. While studying the effect of nanoparticles on biological systems it was found that the effect depended on the particle form. For example, the nanoparticles of AgNP having a triangular form produce more pronounced toxic effect on Escherichia coli than spherical ones [4]. It was also found that breeding sites of solitary bees affected microorganisms at a distance: the viability of yeasts was suppressed (dough inflation was reduced by $26 \%$ ); the same occurred with some saprophytic soil plants (the growth was reduced by $33 \%$ ) [5].

Dowsing is another example of the influence of underground CSs on people $[6,7]$.

The phenomenon of influence of ambient bodies having a CS on biological systems has had so far no established name. In the 1930s De Belizal and Morel [2] proposed the term 'form radiation'. In the classical work of Mermet [8] the term 'radiesthesia' is also used. The Russian scientist Victor Grebennikov (1927-2001) called the phenomenon the 'cavity structure effect' [9]. In this paper the latter term is used, because it reflects most accurately the physical aspect of the phenomenon, i.e. the presence of curvature in the form of the body.

In Europe, Oskar Korschelt (1853-1940) was likely the first who was granted a patent for the use of specially fabricated CSs for medical purposes [10-12]. Figure 1 shows examples of CSs made by Korschelt. The CS in figure 1a is a tin-plate cylinder with soldered zinc or steel teeth. The CS in figure $1 \mathrm{~b}$ is made out of metal wire. The CS shown in figure $1 \mathrm{c}$ is a wooden disk with copper chain coiled on it. The size of each CS does not exceed $50 \mathrm{~cm}$. The $\mathrm{CS}$ used was suspended from the ceiling (fig. 1c) or attached to a floor-type setup (fig. 1d). Using such CSs Korschelt treated stomach problems, nerve diseases, insomnia, and pain.

Korschelt might have gained knowledge about the curative value of such structures in Japan, while attending Tokyo Medical School. Pagodas are tiered buildings with multiple eves, which are common in Japan, China, and many other Eastern countries. They are intended for storing treasures, in particular leather, furs as well as expensive wines, and have been con-

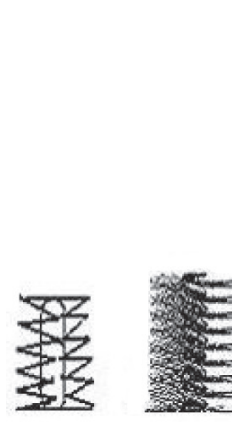

(a)

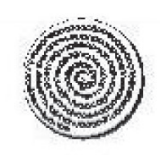

(c)

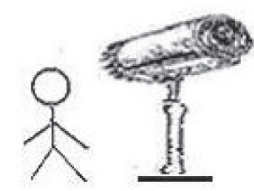

(d)

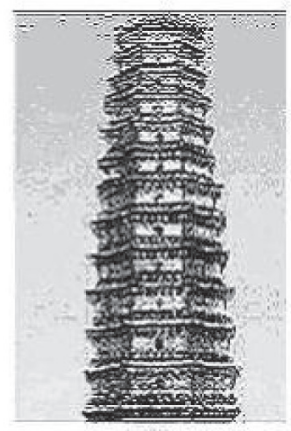

(e)
Fig. 1. The metal CS (a), (b), (c) made out by Korschelt; (d) the device with a metal cavity structure for medical treatment, (e) part of Tie Ta Pagoda (Iron Pagoda), Keifeng (China) in the year 1041.

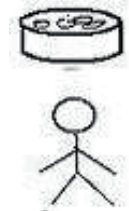

(a)

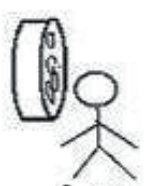

(b)

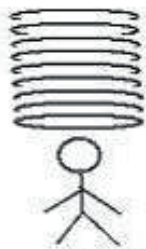

(c)

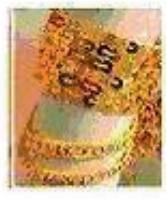

(d)
Fig. 2. Examples of CS used for therapeutic purposes: (a) a sieve put on the head; (b) a sieve held in front of the face; (c) a hoop consisting of wooden rings; (d) bracelets.

structed like CSs (fig. 1e). This shows that since ancient times people have known the unusual properties of such structures.

In Russia the medical aspect of CSs was studied by Grebennikov [5, 9], and there is a museum in Novosibirsk where a device based on empty bee combs is exhibited that was intended for therapeutic purposes. It is noteworthy that in Russia headaches have been treated by a sieve (a cylinder whose bottom is a mesh) for centuries. The sieve was put on the head (fig. 2a) or held in front of the face with the mesh being parallel to the face (fig. 2b). People living on the banks of the lower Amur River used to cure a sick child by putting a hoop on him or her, consisting of wooden rings (fig. 2c) [13]. It is possible that diadems, bracelets, rings, and necklaces were originally used for medical purposes, and only with time they became ornaments (fig. 2d).

The main results obtained in the studies of CS effects on biological objects (BOs) are as follows [5,10,12,13].

1) CSs exert influence on BOs independent of the material the structure is made of: plastics, paper, wood, metal, etc. However, the efficacy of the effect depends on the type of cavity material.

2) The efficacy of the effect depends on the orientation of the $\mathrm{CS}$ with respect to the target $\mathrm{BO}$. For example, the therapeutic effect of the CS oriented as shown in figure $3 \mathrm{a}$ on a human differs from that of the CS oriented as shown in figure $3 b$. 
Fig. 3. Two variants of orientation of a CS (the parts of empty bee combs) with respect to a human in a medical treatment.

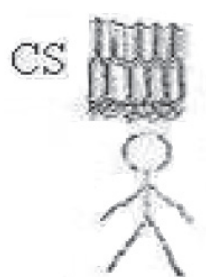

(a)

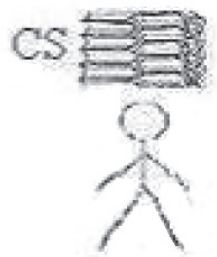

(b)
3) CSs exert their influence on BOs independent of the presence of screens between the CS and the BO, such as brick walls, metal shields, etc

4) The CS influence on the target BO may not cease after removal of the CS. The duration of such after-effect may be some hours or even days.

5) The effects of CSs on people vary from person to person and depend on the state of the person under test. For example, Grebennikov gave the following statistical data on the effects of the empty bee combs: $27 \%$ of people under test felt warmth, $6 \%$ felt cold, $11 \%$ heard various sounds.

It is important to note that the readings of acoustic instruments or thermometers placed near the persons were not changed.

The question arises as to what physical processes determine the specific properties of CSs. For example, Korschelt thought that CSs 'mobilize the vital forces of ether for medical purposes'. Grebennikov maintained that the effect of CS occurred due to the action of a set of de Broglie waves. To the author's knowledge, no physical process in the physical vacuum has been suggested so far for the explanation of CS effects.

To explain the above features of the CS effect on a BO, it is necessary to address the properties of the physical vacuum. A number of works are known in which the physical vacuum is endowed with the properties of the superfluid ${ }^{3} \mathrm{He}-\mathrm{B}$. For example, the superfluid properties of the physical vacuum explain the dissipation-free motion of celestial bodies in space [14]. In different studies [15-18] analogies were revealed between some properties of superfluid ${ }^{3} \mathrm{He}-\mathrm{B}$ and gravitational properties of space. It was also shown that endowing the physical vacuum with the properties of superfluid ${ }^{3} \mathrm{He}-\mathrm{B}$ makes it possible to explain such phenomena as the wave properties of matter, some optical effects, and superconductivity $[19,20]$ This makes it possible to explain the effects of ultra-low doses of biologically active substances on BOs [21].

Under the assumption that the physical vacuum has the properties of superfluid ${ }^{3} \mathrm{He}-\mathrm{B}$ (such physical vacuum will be referred to as the superfluid physical vacuum (SPV)), the specific properties of the $\mathrm{CS}$ effects on a $\mathrm{BO}$ can be taken to be associated with spin supercurrents existing in the CS. From this viewpoint, the CS is 'filled' with spin supercurrents and energy associated with the spin supercurrents, which can be transferred to a BO. The properties of the spin supercurrents are similar to those of spin supercurrents in superfluid ${ }^{3} \mathrm{He}-\mathrm{B}$.

\section{The Mechanism of Action of Cavity Structures on Biological Objects}

\section{The Physical Process Underlying the CS Effect}

According to the SPV model, any quantum entity creates a spin structure in the SPV [20]. Therefore, any body (including biological systems), as consisting of quantum entities, creates spin structures in the SPV.The orientation of the spin structure total spin in space is characterized by 3 angles. If any spin structure has gradients of respective angles, spin supercurrents arise between the structures [22-24]. If a body is flat, the respective spin orientation angles in the spin structures created by the body will have the same values, and there will be no spin supercurrents between the structures. If the body contains a cavity, i.e. it is a CS, the respective spin orientation angles in the spin structures created by the body cannot be equal and thus there will be spin supercurrents between them, i.e. the cavity will be 'filled' with spin supercurrents.

As follows from the definition of the spin supercurrent [23, 24], the magnitude of the latter depends on the characteristics of spin structures, between which the spin supercurrent arises, i.e. it depends on the material of which the CS is made.

Since spin supercurrents arise between the spin structures produced by bodies of any type, including BOs, they would also arise between spin structures produced in the SPV by CSs and BOs. The spin supercurrents determine the effect of the $\mathrm{CS}$ on the $\mathrm{BO}$.

\section{The Dependence of Efficacy of the CS on Its Orientation with Respect to the $B O$}

Since spin supercurrents are determined by the gradients of spin orientation angles in space, different orientation of the CS with respect to the $\mathrm{BO}$ may result in different therapeutic efficacy.

\section{The Independence of Efficacy of CS of the Presence of Screens}

Spin supercurrents by which the BO and CS interact exist in a 'finer' physical medium (the physical vacuum) than the molecular one. Therefore, the spin supercurrents may not be shielded by molecular substances.

\section{The After-Effect of CS}

The spin structures created by the CS in the SPV form a ring. According to the SPV model, the ring of spin structures is a quantum vortex ring in the SPV [20]. In the stationary state the quantized vortices in the superfluid do not diffuse and, consequently, will not disappear after removing the object that has caused them from the region of space it occupied. Thus after removing the CS from the region of space it occupied, a 'trace' of the CS may remain there in the form of vortex rings in the SPV. 


\section{Sensations Experienced by Humans - the Problem of Measurement}

According to the SPV model, the value of a spin supercurrent depends on the characteristics of the spin structures between which the current arises, and, consequently, on the properties of the bodies that produce the spin structures in the SPV. If the BO is a human, the sensations he or she experiences depend on the organs the CS exerts action upon and on the organs that are affected. As a result, the human may experience various sensations such as warmth, cold, or various sounds.

If an instrument is placed near the CS to measure temperature or acoustic vibrations, the characteristics of the spin structures produced by the instrument will generally be different from those of the spin structures produced by the BO. Consequently, spin supercurrents arising between the CS and BO will be different from spin supercurrents arising between the CS and the instrument. Thus in assessing the effects produced by CSs the problem of measurement is no less acute than that in measuring the characteristics of quantum objects in conventional physical experiments. While in the latter case, under the uncertainty principle, there is uncertainty in determining some characteristics of the objects, in assessing the CS effects on the BO the uncertainty extends even on the type of the effects observed.

\section{Discussion}

In several publications [15-18] analogies are drawn between some properties of superfluid ${ }^{3} \mathrm{He}-\mathrm{B}$ (described by the order parameter) and gravitational properties of space. At the same time the spin supercurrents inside a CS are determined by a gradient of order parameter phase in the SPV. Consequently, it could be assumed that the gravitational properties of the physical vacuum change in the vicinity of CS. For example, it is known that the cocoon of an ichneumon of the Ichneumonidae family belonging to Bathyplectes anurus species (the cocoon being a CS) jumps upon exposing it to sunlight. The jumps are $30 \mathrm{~mm}$ long and $50 \mathrm{~mm}$ high, exceeding the cocoon width by a factor of 30 [25]. In Grebennikov's experiments such jumps were performed even when the cocoon had been placed on a 'cloud' of loose cotton wool [9]. The gravitational properties of space affect propagation of light. Therefore, optical effects can be observed near CSs. For example, Korschelt observed weak illumination of CSs in the dark. Grebennikov observed short-term multiple 'disappearance' of the Bathyplectes anurus cocoon during its jumps [9]. Thus CS energy properties could be measured by sensitive gravimeters or devices detecting the laser beam deviation near the CS.
While analyzing human environmental conditions it is necessary to take into account the possibility of both positive and negative effects of ambient structures on human beings. In particular, people are affected by the form of pots and pans, headwear, necklaces, pieces of furniture, rooms, buildings, etc. According to the above mentioned CS properties, the efficacy of a CS does not depend on the presence of screens; therefore, a human being is affected also by underground facilities, such as tunnels, utility galleries, underground garages, metro, etc.

To determine the most favorable forms of ambient bodies for humans, it is necessary, first, to carry out complex analysis of spin supercurrents arising in CSs and, second, to experimentally investigate how spin supercurrents affect the human organism. However, some recommendations can be given at the stage of CS effect investigations: one should not have one's sleeping accommodation located in such a way that one's head would be in a corner of the premises; one should not stay long in the premises which are a sort of narrow niches, or in a deep armchair with a metal frame (according to the SPV model, spin currents may be greater in metals). The following way of self-healing may be used: laying the palms of hands on a problem area of the body so that the fingers of one hand are at an angle to the fingers of the other hand, thus forming a grate.

The key question is that of shielding the effects of CSs. De Belizal and Morel [2] described experiments where the effect shielding of a CS set, i.e. coaxially joined hollow-wooden hemispheres, was obtained using several layers of specially fabricated polyethylene film. According to the SPV model, the irradiation of a CS by light has to affect its properties, because photons are quantum entities that produce spin structures in the physical vacuum, that interact with the spin structures produced by the CS. Thus the effect of a CS on ambient bodies in the dark will be different from that in broad daylight.

\section{Conclusion}

The assumption that the physical vacuum has properties of superfluid ${ }^{3} \mathrm{He}-\mathrm{B}$ would mean that every cavity would be filled with spin supercurrents. The way these would interact with biological matter can explain many of the observed qualitative effects of CSs on the human body. However, designing instruments to measure such spin supercurrents is fraught with difficulties, such as the existence of after-effects and the effect of the material of the instrument on the spin supercurrents to be measured.

\section{Disclosure Statement}

The author declares that there is no conflict of interest concerning this paper. 


\section{References}

1 Dimde M: Die Heilkraft der Pyramiden. München, Heyne, 2000.

2 De Belizal A, Morel PA: Physique Micro-Vibratoire et Forces Invisibles. Paris, Edition Desforges, 1965

3 Pagot J: Radiestesie et Emission de Form. Maloine, Paris, 1978.

4 Pal S, Tak YK, Song JM: Does the antibacteria activity of silver nanoparticles depend upon the shape of the nanoparticles? Appl Environ Microb 2007;73:1712

5 Grebennikov VS: The secret of breeding sites of solitary bees (in Russian). Beekeeping 1984;12:28-29.

6 Betz H-D: Unconventional water detection: field test of the dowsing technique in dry zones: part 1. J Sci Explor 1995;9:1-43.

7 Betz H-D: Unconventional water detection: field test of the dowsing technique in dry zones: part 2. J Sci Explor 1995;9:159-189.

8 Mermet A: Principles and Practice of Radiesthesia. New York, Harper/Collins, 1959.

9 Grebennicov VS: My World (in Russian). Novosibirsk, Sovetskaya Sibir Publisher, 1997. (Chapter V of this book has been translated into English by Dr Cherednichenko: www.keelynet.com/greb/greb.htm (accessed on 23.09.2013)).
10 Kaiserliches Patentamt, Patentschrift Nr. 69340, ausgegeben am 22,6,1893 Klasse 30: Gesundheitspflege. Oskar Korschelt in Leipzig. Ein Apparat für therapeutische Zwecke ohne bestimmte oder bewußte Suggestion. Patentiert im Deutschen Reiche vom 14. Juni 1891.

11 Korschelt O: Die Nutzbarmachung der lebendigen Kraft des Äthers in der Heilkunst, Landwirtschaft und Technik, ed 2. Leipzig, Verlag F.E. Baumann, 1921.

12 Schrödter W: Planzengeheimnisse, Kap. 'Pflanzen als Strahlapparate', ed 3. Kleinjörl bei Flensburg, G.E. Schroeder-Verlag, 1981.

13 Smolyak AV: Shaman: The Personality, Functions, Way of Thinking (the People of Lower Amur) (in Russian). Moscow, Nauka, 1991.

14 Sinha KP, Sudarshan ECG: The superfluid as a source of all interactions. Found Phys 1978;8:823831.

5 Ruutu VMH, Eltsov VB, Gill AJ, et al.: Vortex formation in neutron-irradiated superfluid ${ }^{3} \mathrm{He}$ as an analog of cosmological defect formation. Nature 1996;382:334-336.

16 Eltsov VB, Kibble TW, Krusius M, et al.: Composite defect extends analogy between cosmology and ${ }^{3}$ He. Phys Rev Lett 2000;85:4739-4742.
17 Volovic GE: The Universe in a Helium Droplet. Oxford, Clarendon Press, 2003.

18 Winkelmann CB, Elbs J, Bunkov YM, Godfrin $\mathrm{H}$ : Probing 'cosmological' defects in superfluid ${ }^{3} \mathrm{He}-\mathrm{B}$ with a vibrating-wire resonator. Phys Rev Lett 2006; 96:205301.

19 Boldyreva LB, Sotina NB: Superfliud vacuum with intrinsic degrees of freedom. Physics Essays 1992;5: 510-513.

20 Boldyreva LB: What Does This Give to Physics: Attributing the Properties of Superfluid ${ }^{3} \mathrm{He}-\mathrm{B}$ to Physical Vacuum? Moscow, Krasand, 2012.

21 Boldyreva LB: An analogy between effects of ultra low doses of biologically active substances on biological objects and properties of spin supercurrents in superfluid ${ }^{3} \mathrm{He}$-B. Homeopathy 2011;100:187-193.

22 Borovic-Romanov AS, Bunkov YM, Dmitriev VV, et al.: Investigation of spin supercurrents in ${ }^{3} \mathrm{He}-\mathrm{B}$. Phys Rev Lett 1989;62:1631.

23 Dmitriev VV, Fomin IA: Homogeneously precessing domain in ${ }^{3} \mathrm{He}-\mathrm{B}$ : formation and properties. J Phys Condens Matter 2009;21:164202.

24 Bunkov YM: Spin superfluidity and coherent spin precession. J Phys Condens Matter 2009;21:164201.

25 Hymenopterans. Ichneumonidae; in Medvedev GS (ed): The Ranger of Insects in the European Part of the USSR. Leningrad, Nauka 1981, vol. III, part 3:26. 